\title{
Unexpected differences between surface and bulk spectroscopic and implied Kondo properties of heavy fermion $\mathrm{CeRh}_{2} \mathrm{Si}_{2}$
}

Georg Poelchen (iD ${ }^{1,2 凶}$, Susanne Schulz ${ }^{1}$, Max Mende ${ }^{1}$, Monika Güttler ${ }^{1}$, Alexander Generalov ${ }^{3}$, Alexander V. Fedorov ${ }^{4}$, Nubia Caroca-Canales ${ }^{5}$, Christoph Geibel $\mathbb{D}^{5}$, Kristin Kliemt $\mathbb{D}^{6}$, Cornelius Krellner $\mathbb{1}^{6}$, Steffen Danzenbächer ${ }^{1}$, Dmitry Yu. Usachov ${ }^{7}$, Pavel Dudin ${ }^{8,9}$, Victor N. Antonov ${ }^{10}$, James W. Allen ${ }^{11}$, Clemens Laubschat ${ }^{1}$, Kurt Kummer $\left(\mathbb{D}^{2}\right.$, Yuri Kucherenko ${ }^{10}$ and Denis V. Vyalikh (iD ${ }^{12,13 凶}$

Ultra-violet angle-resolved photoemission spectroscopy (UV-ARPES) was used to explore the temperature dependence of the Ce-4f spectral responses for surface and bulk in the antiferromagnetic Kondo lattice $\mathrm{CeRh}_{2} \mathrm{Si}_{2}$. Spectra were taken from Ce- and $\mathrm{Si}$ terminated surfaces in a wide temperature range, and reveal characteristic $4 f$ patterns for weakly (surface) and strongly (bulk) hybridized $\mathrm{Ce}$, respectively. The temperature dependence of the Fermi level peak differs strongly for both cases implying that the effective Kondo temperature at the surface and bulk can be rather distinct. The greatly reduced crystal-electric-field (CEF) splitting at the surface gives reason to believe that the surface may exhibit a larger effective Kondo temperature because of a higher localmoment effective degeneracy. Further, the hybridization processes could strongly affect the $4 f$ peak intensity at the Fermi level. We derived the $k$-resolved dispersion of the Kondo peak which is also found to be distinct due to different sets of itinerant bands to which the $4 f$ states of surface and bulk Ce are coupled. Overall our study brings into reach the ultimate goal of quantitatively testing many-body theories that link spectroscopy and transport properties, for both the bulk and the surface, separately. It also allows for a direct insight into the broader problem of Kondo lattices with two different local-moment sublattices, providing some understanding of why the cross-talking between the two Kondo effects is weak.

npj Quantum Materials (2020)5:70; https://doi.org/10.1038/s41535-020-00273-7

\section{INTRODUCTION}

Rare-earth (RE) intermetallics with an unstable valence form a prototype of strongly correlated electron systems, where the correlations arise from the interplay between almost localized $4 f$ electrons and itinerant valence-band (spd) states. They lead to a wealth of extraordinary phenomena like the formation of ultraheavy quasiparticles (heavy fermions), unconventional superconductivity, magnetic order, and non-Fermi liquid ground states, that may even be degenerate with each other at quantum critical points of their phase diagrams ${ }^{1-6}$.

In cerium materials ${ }^{7}$, the dual nature (localized and itinerant) of the $4 f$ electron is manifested in its most pronounced form leading to the whole range of the aforementioned phases. With the help of angle-resolved photoemission spectroscopy (ARPES) in the vacuum ultra-violet (VUV) regime, it is possible to gain insight into the peculiarities of the $f-d$ interplay and to explore its temperature $(T)$-dependent properties ${ }^{7-9}$. The Ce- $4 f$ emissions reveal a characteristic structure with a feature at a binding energy (BE) of about $2 \mathrm{eV}$ corresponding roughly to a $\mathrm{Ce}-4 f^{0}$ final state and a spin-orbit (SO) split structure at the Fermi energy $\left(E_{\mathrm{F}}\right)$ exhibiting mainly $4 f^{1}$ character. The appearance of the latter is a hybridization effect making this feature sensitive to the valence-band structure and crystal electric field (CEF). For this reason, the $4 f^{1}$ emission attracts particular interest, consisting of a narrow peak at $E_{\mathrm{F}}$, which is attributed to the tail of the Kondo resonance lying slightly above $E_{\mathrm{F}}{ }^{7}$, and its satellites ${ }^{7-11}$.

Due to the small mean free path of the photoelectrons, VUVARPES is a strongly surface-sensitive technique which makes studies of bulk properties an intricate task. At the surface, the atomic coordination changes and at the same time the valenceband structure can be strongly modified by surface states and relaxation effects. In mixed-valent heavy RE compounds, this usually leads to a surface valence transition to a stable divalent configuration $^{12}$. However, Ce systems also remain mixed valent at the surface due to a strong $4 f$ hybridization. But because of the reduced atomic coordination at the surface, the hybridization strength is decreased, resulting in a transition from strongly ( $a$ like) to weakly hybridized ( $\gamma$-like) Ce at the surface ${ }^{10,13,14}$.

For a correct interpretation of ARPES spectra, these different contributions from surface and bulk need to be separated. Foremost, a high sample quality and careful surface preparation are needed as the cleavage of single crystals usually results in the formation of surfaces with different terminations ${ }^{7,10,11,15}$. Even then, distinguishing surface and bulk of $\mathrm{Ce}-4 f$ spectra is not trivial, since the corresponding contributions occur at the same $\mathrm{BE}^{13,14}$. Possible approaches to separate bulk and surface contributions are (i) the quenching of the latter by deposition of adlayers ${ }^{16}$ or (ii) the tuning of surface sensitivity by variation of the photon energy or electron emission angle ${ }^{17}$. However, while the use of adlayers

\footnotetext{
${ }^{1}$ Institut für Festkörper- und Materialphysik, Technische Universität Dresden, D-01062 Dresden, Germany. ${ }^{2}$ European Synchrotron Radiation Facility, 71 Avenue des Martyrs, F-38043 Grenoble, France. ${ }^{3}$ Max IV Laboratory, Lund University, Box 118, SE-22100 Lund, Sweden. ${ }^{4}$ IFW Dresden, POB 270116 , D-01171 Dresden, Germany. ${ }^{5}$ Max Planck Institute for Chemical Physics of Solids, Nöthnitzer Str. 40, D-01187 Dresden, Germany. ${ }^{6}$ Kristall- und Materiallabor, Physikalisches Institut, Goethe-Universität Frankfurt, Max-von-Laue Strasse 1, D-60438 Frankfurt am Main, Germany. ${ }^{7}$ St. Petersburg State University, 7/9 Universitetskaya nab., 199034 St. Petersburg, Russia. ${ }^{8}$ Diamond Light Source, Harwell

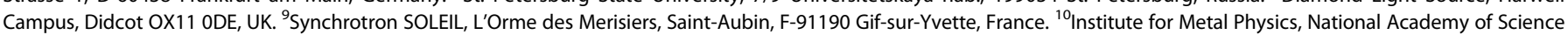
of Ukraine, UA-03142 Kiev, Ukraine. ${ }^{11}$ Randall Laboratory, University of Michigan, 450 Church St., Ann Arbor, MI 48109-1040, USA. ${ }^{12}$ Donostia International Physics Center (DIPC), 20018 Donostia-San Sebastián, Spain. ${ }^{13}$ IKERBASQUE, Basque Foundation for Science, 48011 Bilbao, Spain. ${ }^{凶}$ email: georg.poelchen@tu-dresden.de; denis.vyalikh@dipc.org
} 
involves the risk of changing the structure and chemical composition of the near-surface region, variations of photon energy and emission angle change the cross sections and the position in $k$ space making the reliability of these procedures questionable. Thus, investigating the temperature-dependent properties of Ce systems is a complicated task and the mentioned issues need to be considered.

In that regard, the antiferromagnetic (AFM) Kondo lattice $\mathrm{CeRh}_{2} \mathrm{Si}_{2}\left(T_{\mathrm{N}}=38 \mathrm{~K}, T_{\mathrm{K}} \sim 30 \mathrm{~K}\right)^{18-21}$ seems to be an ideal candidate $^{10}$. This compound crystallizes in the body-centered tetragonal $\mathrm{ThCr}_{2} \mathrm{Si}_{2}$ type structure (14/mmm space group, No. 139), in which layers of $\mathrm{Ce}$ atoms are well separated by $\mathrm{Si}-\mathrm{Rh}-\mathrm{Si}$ trilayers. With a much stronger bonding between the Si and Rh than Si and $\mathrm{Ce}$ atoms, the samples predominantly cleave between the $\mathrm{Si}$ and Ce layers. We have shown previously ${ }^{10}$ that the resulting $\mathrm{Ce}$ - or Siterminated surfaces exhibit strongly different $4 f$ spectral functions at $1 \mathrm{~K}$ corresponding to the paradigmatic response of weakly or strongly hybridized $\mathrm{Ce}$. While for the Ce-terminated surface more than $60 \%$ of the $4 f$ emissions originate from the surface Ce atoms, the $4 f$ emissions from the Si-terminated surface stem predominantly from Ce atoms located in the fourth subsurface layer and thus reflect bulk-like properties.

Because the two surfaces can be measured individually on the same sample, $\mathrm{CeRh}_{2} \mathrm{Si}_{2}$ offers the opportunity to directly and simultaneously probe the differences for surface and bulk $\mathrm{Ce}$ atoms of the momentum- and temperature-dependent properties of the $f-d$ hybridization. The most essential findings are the clear demonstration of surprising differences between surface and bulk for (i) the temperature dependence of the $4 f$ spectral pattern near $E_{\mathrm{F}}$ and (ii) the momentum dependence of the Kondo resonance, especially above $E_{\mathrm{F}}$. We show that the first of these can be understood as resulting from a much reduced CEF splitting on the surface relative to that of the bulk, and that it has a further implication that is unexpected based on previous works. The second can be traced to different surface and bulk conductionband electronic structures identified in ab initio band-structure calculations in the framework of density functional theory (DFT). The results are enabled by an analysis procedure that yields more detailed insight into the properties of the states above $E_{\mathrm{F}}$ than could be achieved by the standard approach of division by a resolution-convolved Fermi-Dirac function. Although this procedure is inspired and required by the particular situation, it may well be more generally applicable. Overall our study brings into reach the ultimate goal of quantitatively testing many-body theories that link spectroscopy and transport properties, for both the bulk and the surface, separately. It also allows for a direct insight into the broader problem of Kondo lattices with two different local-moment sublattices, providing some understanding of why the cross-talking between the two Kondo effects is weak.

\section{RESULTS AND DISCUSSION}

\section{Description of ARPES data}

In Fig. 1, we present ARPES data taken from Ce- and Si-terminated surfaces of $\mathrm{CeRh}_{2} \mathrm{Si}_{2}$ along the $\bar{M}-\bar{\Gamma}-\bar{M}$ direction. The data are centered around the $\bar{\Gamma}$ point in an interval of $\pm 4^{\circ}$ while $\bar{M}$ would be situated at an emission angle of $11.4^{\circ}$. The corresponding angleintegrated photoemission (PE) signals over the presented angle interval are shown in the lower panels. The $4 f$ signal is dominated by a sharp peak at $E_{\mathrm{F}}$ which stems from $C e-4 f_{5 / 2}^{1}$ emissions and contains the main information about the Kondo physics of the system. In addition, at the Ce-terminated surface, a $4 f_{7 / 2}^{1}$ SO satellite is seen at about $-300 \mathrm{meV}$ in accordance with the wellknown Ce atomic SO splitting of $285 \mathrm{meV}$. In the bulk (Siterminated surface), its BE is reduced to about $250 \mathrm{meV}$ reflecting either an effect of hybridization with the valence bands ${ }^{22}$ or increased screening of the core potential ${ }^{23}$.
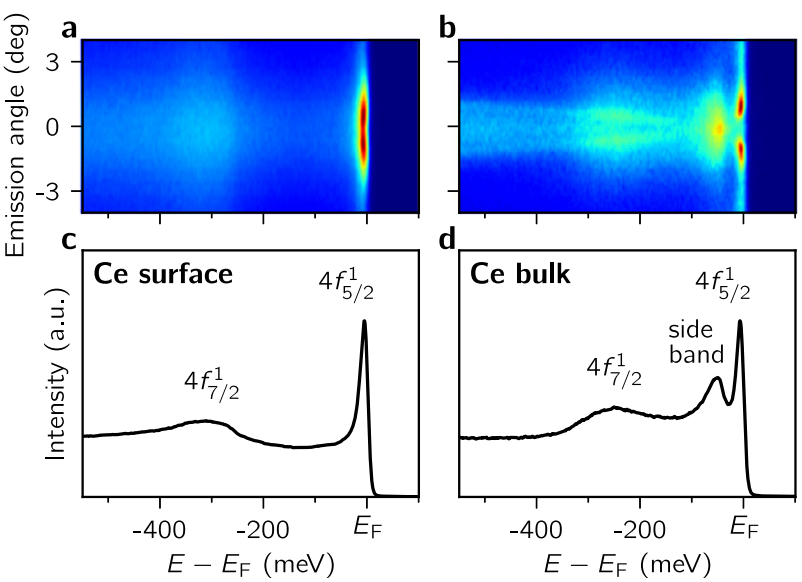

Fig. 1 Spectral structure of the surface terminations of $\mathrm{CeRh}_{2} \mathrm{Si}_{2}$. ARPES view on the Ce- $4 f$ electronic structure around the $\bar{\Gamma}$ point at $7 \mathrm{~K}$ for $\mathbf{a} \mathrm{Ce}$ - and $\mathbf{b}$ Si-terminated surfaces of $\mathrm{CeRh}_{2} \mathrm{Si}_{2}$ taken with a photon energy of $121 \mathrm{eV}$. Corresponding PE spectra obtained upon integration over the shown interval of -4 to $4^{\circ}$ (corresponding to a $k$ segment from -0.38 to $0.38 \AA^{-1}$ at $E_{\mathrm{F}}$ ) are shown in (c, d). Strong shift of the $4 f_{7 / 2}^{1}$ feature as well as the appearance of a sideband from the main $4 f_{5 / 2}^{1}$ peak at about $-50 \mathrm{meV}$ indicates two distinct terminations.

Further, considering the nature of a sideband at about $-50 \mathrm{meV}$ in the bulk spectrum, we have to emphasize that this feature is determined by two contributions. First, in the bulk, the $D_{4 h}$ symmetry leads to a splitting of the ${ }^{2} F_{5 / 2}$ state into three Kramers doublets $\left(\Gamma_{6}\right.$ and two $\left.\Gamma_{7}\right)$ with a CEF scheme determined by inelastic neutron scattering and soft X-ray absorption spectroscopy ${ }^{24}$ as well as high-resolution resonant inelastic soft X-ray scattering ${ }^{25}$ to be a $\Gamma_{7}$ ground state with the first and second excited states around 30 and $53 \mathrm{meV}$. The $4 f$ CEF-split states were recently observed in $\mathrm{ARPES}^{10}$, where a CEF scheme of 0-48-62 meV was established. Here they are visible in Fig. $2 \mathrm{~d}$ as low-intensity weakly dispersive structures. Second, the sideband shows a large intensity at the $\bar{\Gamma}$ point. This is the result of the hybridization of the Ce- $4 f_{5 / 2}^{1}$ state with a hole-like valence band approaching $E_{\mathrm{F}}$. This leads to a characteristic redistribution of the $4 f^{1}$ emission at the $\bar{\Gamma}$ point ${ }^{26}$ : the $f-d$ hybrid states with a significant $f$ contribution are created at the top of the valence band whereas a strong decreasing of $4 f^{1}$ intensity is observed at $E_{\mathrm{F}}$ due to the shift of the Kondo peak toward higher energies (as discussed below).

Although the CEF scheme for the bulk is known ${ }^{10}$, for the Ceterminated surface no formations of CEF-split bands have been experimentally found so far. Comparing the normalized spectra of the Ce- and Si-terminated surfaces (Fig. 2b-d), the missing sideband of the Ce-terminated surface is especially apparent. It could be expected that on the surface the CEF splittings are too small to be resolved (with an energy resolution of around $10 \mathrm{meV}$ at a photon energy of $121 \mathrm{eV}$ ). Such strong changes can be understood as a result of symmetry breaking at the surface. In the bulk, the CEF is characterized by weak components "in plane" ( $a b$ plane) due to the similarly charged Ce-neighbor atoms and a strong component perpendicular (along the $c$ axis) caused by the polar bonds to the Si-Rh-Si triple layer. Expanding the component along the $c$ axis in $z$ direction as power series $V_{\mathrm{CEF}}(z) \sim \sum a_{i} z^{j}$, only terms with even $i$ contribute to the series due to the underlying $D_{4 h}$ mirror symmetry $\left(a_{i}=0\right.$ for all odd $\left.i\right)$. Thus, the mirrorsymmetric $V_{\mathrm{CEF}}(z)$ leads in first-order perturbation theory to $4 f$ energy splittings different from zero. However, at the surface the $D_{4 h}$ symmetry is reduced to a $C_{4 v}$ symmetry and the mirrorsymmetric $V_{\mathrm{CEF}}(z)$ is replaced by an asymmetric dipole field along the $c$ axis. The resulting power series expansion would thus be 

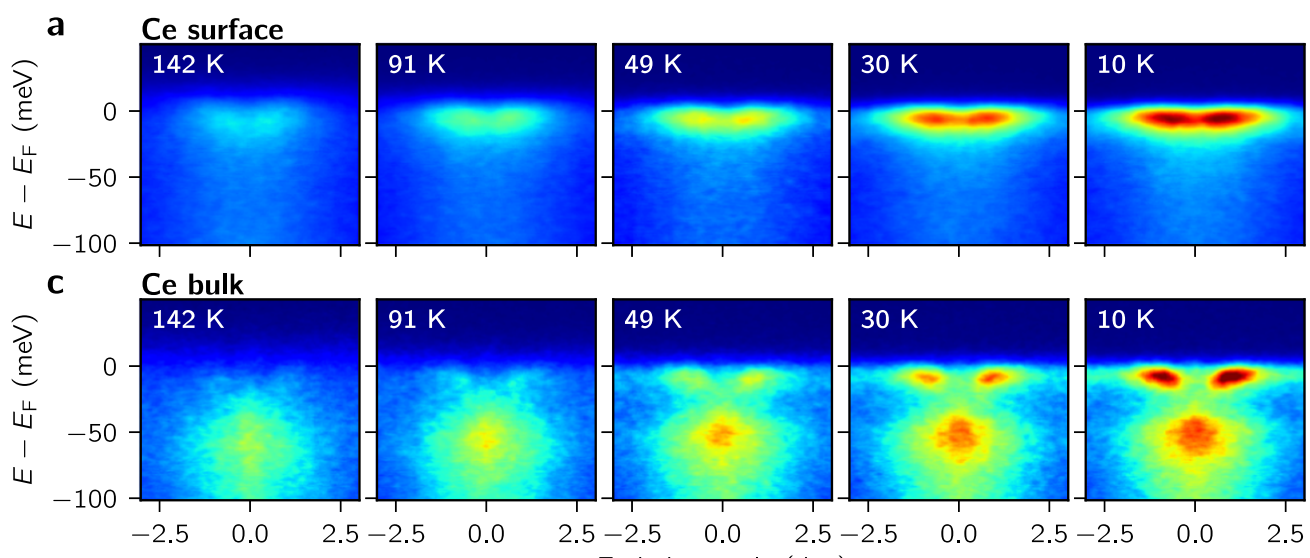

Emission angle (deg) b
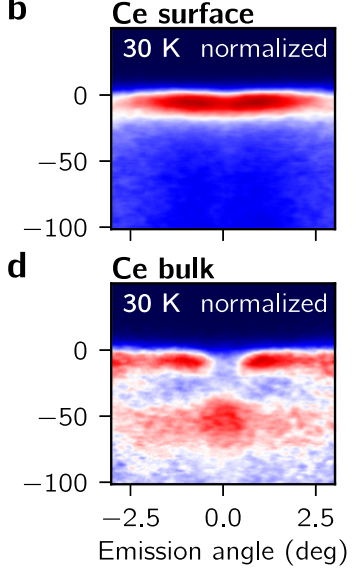

Fig. 2 Temperature-dependent view of the ARPES measurements. Temperature dependence of the $k$-resolved Ce- $4 f$-derived states near the $\bar{\Gamma}$ point for a Ce- and c Si-terminated surfaces of $\mathrm{CeRh}_{2} \mathrm{Si}_{2}$. The ARPES data were taken along the $\bar{M}-\bar{\Gamma}-\overline{\mathrm{M}}$ direction of the surface Brillouin zone (SBZ). b, $\mathbf{d}$ Normalization of each angle slice to the same integral intensity of the $30 \mathrm{~K}$ spectra in order to enhance weak contributions of the $4 f C E F$ to the sideband.

dominated by odd exponents in $i$ (with prefactors $a_{i}$ being small or zero for even $i$ ). This would cause in first-order perturbation theory the disappearance or at least strong decrease of the energy splittings (for an odd potential, meaning $a_{i}=0$ for all even $i$, $\left|\left\langle V_{\mathrm{CEF}}(z)\right\rangle\right|^{2} \sim\left|\sum_{\text {iodd }} a_{i}\left\langle\psi\left|z^{i}\right| \psi\right\rangle\right|^{2}=0$ for a non-degenerate atomic ground state $\Psi$ ) and leading to the observed collapse of the CEF splitting.

That such a loss of mirror symmetry has indeed a dramatic effect on the crystal electric field is experimentally demonstrated by the case of the $R E \mathrm{Ir}_{2} \mathrm{Si}_{2}$ compounds. These compounds can crystallize in two different, but closely related structure types, the $\mathrm{ThCr}_{2} \mathrm{Si}_{2}$ body-centered type (called l-type) as for $\mathrm{CeRh}_{2} \mathrm{Si}_{2}$, and the $\mathrm{CaBe}_{2} \mathrm{Ge}_{2}$ type (called P-type). In the latter one, the position of $\mathrm{Si}$ and $\mathrm{Ir}$ are interchanged in every second $\mathrm{Ir}-\mathrm{Si}$ layer. This leads to the loss of the $c$-axis mirror and inversion symmetry at the Ce site, while the number of $\mathrm{Ir}$ and $\mathrm{Si}$ nearest neighbors to Ce remains unchanged. The loss of mirror symmetry results in a strong change in the crystal electric field: let us take, e.g., the case of $\mathrm{Dylr}_{2} \mathrm{Si}_{2}{ }^{27}$. The leading CEF coefficient has a different sign in the $\mathrm{I}-$ and $\mathrm{P}$ type, resulting in a change from a strong Ising magnetic anisotropy in the I-phase to a $X Y$ anisotropy in the P-type. Simultaneously the overall CEF splitting decreases from $500 \mathrm{~K}$ in the I-type to $300 \mathrm{~K}$ in the P-type. Since the difference in the surrounding between $\mathrm{Ce}$ in the bulk and $\mathrm{Ce}$ at the surface (half of the ligands are missing on one side) is much stronger than the difference between the Ce surrounding in I- and P-type (same number of ligands on both sides), one has to expect a much stronger difference in the CEF scheme and CEF splitting in our case. Thus in view of the experimental observation of strong differences in the CEF scheme and CEF splitting between I- and Ptype $R E \mathrm{Ir}_{2} \mathrm{Si}_{2}$, it is not surprising that such a strong change between surface and bulk can be expected.

Temperature dependence and its interpretation

The Kondo temperature is usually determined theoretically from photoemission data by estimating the $4 f$ hybridization as being defined in the single-impurity Anderson model (SIAM) from the intensity ratio of the $4 f^{1}$ emission at $E_{\mathrm{F}}$ and the $4 f^{0}$ emission at a $\mathrm{BE}$ of about $2 \mathrm{eV}$. However, this method is not applicable to the present angle-resolved photoemission data, since the $4 f^{1}$ emission at $E_{\mathrm{F}}$ varies strongly as a function of the wave vector $k$ due to hybridization with the dispersive valence bands and an integral measurement over the entire Fermi surface is not possible. In the following, we will therefore try to determine the Kondo temperature for surface and bulk from the observed temperature dependence of the Kondo resonance. Starting point is the $4 f k$ resolved electron landscape which is changed as a function of $T$ as shown in Fig. $2 \mathrm{a}$, c. It can be seen that the $4 f$ spectral pattern reveals well distinguishable modifications for both surface terminations when the temperature is lowered. The $k$-dispersive $4 f$ structure at $E_{\mathrm{F}}$ is almost not observable at $142 \mathrm{~K}$, but gradually enhances with falling temperature becoming most intense at $10 \mathrm{~K}$. Interestingly, the sideband around $-50 \mathrm{meV}$ reveals a similar, but weaker $T$ dependence and remains visible at $142 \mathrm{~K}$.

We analyzed these data as described next. We note some similarities to an analysis of $T$-dependent ARPES data for $\mathrm{CeColn}_{5}{ }^{28}$. As the first step, we performed an angle integration of the ARPES data in a window of $\pm 2^{\circ}$ around the $\bar{\Gamma}$ point $(k$ segment of $\pm 0.19 \AA^{-1}$ at $E_{\mathrm{F}}$ ). The $T$ dependence of the $4 f$ signal, derived in this way for each termination of the crystal, is shown in Fig. 3. The measurements show for each termination a clear, but noticeably different, decrease of the $E_{\mathrm{F}}$ peak with increasing $T$. To characterize this behavior, we further performed a background subtraction for each spectrum in Fig. 3a, b. Note that subtraction of the Fermi-Dirac distribution (FDD), of a linear background, and of the highest- $T$ spectrum, were all tested and lead to similar results (see Supplementary Discussion 1). The black dots in Fig. 3c, d correspond to the integrated intensities around the $E_{\mathrm{F}}$ peak after subtraction of the FDD background.

The $T$ dependences of these intensities can be nicely fitted by an exponential function of the form $\exp -\frac{T}{T_{0}}$. Note, that the Kondo behavior is generally associated with the logarithmic $\frac{T}{T_{\mathrm{K}}}$ dependence (for a comparison see Supplementary Discussion 2). However, the use of an exponential function is motivated by the fact that for many properties of Kondo systems the logarithmic behavior occurs only over a limited $T$ range. The full $T$ dependence, the logarithmic region and the departures from it at high and low $T$, is much better described as exp $-\frac{\pi T}{T_{\mathrm{K}}}$ (see details in Supplementary Discussions 3). Because we only consider a region near the high-symmetry point, a theoretical description in the framework of the SIAM is not applicable and a direct inference of $T_{\mathrm{K}}$ from $T_{0}$ is not justified. However, we expect both temperatures to be closely related and we note that for CeColn ${ }_{5}^{28}$, the $T$ dependence of the ARPES $E_{\mathrm{F}}$ peak at a specific point in $k$ space was qualitatively similar to what is found here and compared well with the $T$ dependence of the $E_{\mathrm{F}}$ peak of the $k$ integrated spectrum of a calculation within dynamical mean-field theory. Thus we take the difference in $T_{0}$ to reflect a change of $T_{\mathrm{K}}$.

Experience from early PE studies of polycrystalline samples ${ }^{7}$ is that $T_{\mathrm{K}}$ for the surface is always smaller than for the bulk. This is 

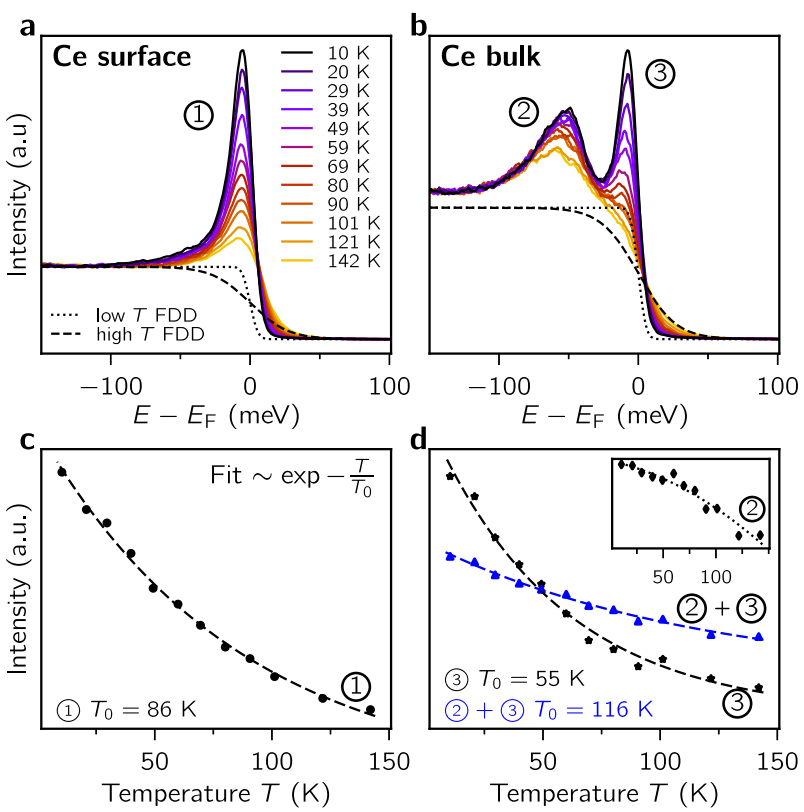

Fig. 3 Temperature-dependent behavior of the integrated intensities of the $E_{\mathrm{F}}$ peak. $\mathrm{PE} k$-integrated profiles of the $4 f$-derived states for various $T$ taken from $\mathbf{a}$ Ce- and $\mathbf{b}$ Si-terminated surfaces of $\mathrm{CeRh}_{2} \mathrm{Si}_{2}$ and the respective $T$ dependence of the $4 f_{5 / 2}^{1}$ peak intensity in (c, d). The integral intensity of the PE peaks was obtained from the data shown in $(\mathbf{a}, \mathbf{b})$ after subtraction of the $T$-dependent FDD energy profiles (convolved with Gaussian resolution broadening). The $T$ dependences in (d) were obtained for the integral intensity of the peak (3) at $E_{\mathrm{F}}$ as well as for the total intensity of both split peaks $(2+3)$. The total intensity of peaks $(2+3)$ in blue was scaled by a factor of $1 / 4$ to directly compare the $T$ behavior to peak (3) in black dots. Inset: the $T$ dependence of the integral intensity of the peak (2) alone.

understood as a consequence of the surface reduction in atomic coordination, which leads both to a reduction of the hybridization strength $V$ of $4 f$ states with valence bands and an increase of the $4 f$-electron BE $\epsilon_{f}$, causing a decrease of the Kondo coupling constant $J \propto \frac{\left(N_{f}\right)\left(V^{2}\right)}{\epsilon_{f}}$, where $N_{f}$ is the ground-state degeneracy ${ }^{7}$. However, the obtained $T$ dependences of the $E_{\mathrm{F}}$ peaks shown in Fig. $3 c$, d suggest the opposite conclusion, i.e. the characteristic temperature $T_{0}$ of the bulk emission $(\sim 55 \mathrm{~K})$ is by a factor of one and a half smaller than that of the surface ( $~ 86 \mathrm{~K})$.

As we now elaborate, the new and unexpected difference in the surface and bulk values of $T_{0}$ can be traced to the reduction of the surface CEF splittings, pointed out already above. We do not know the exact values of the surface CEF splittings but they are certainly less than our energy resolution $\approx 10 \mathrm{meV}=116 \mathrm{~K}$. Within that framework we discuss two limiting cases.

For the first case we simply note that, whatever the splittings are, the $E_{\mathrm{F}}$ peak intensity that we measure certainly includes that of the Kondo resonance and the CEF sidebands. Thus, for the surface spectrum, there is an increase of the effective degeneracy to $N_{f}=6$ relative to that in the bulk spectrum $\left(N_{f}=2\right)$. We propose that the increase of $N_{f}$ overcomes the effects of the decreased surface coordination so that the net effect is an increase of $J$, leading to an increase of $T_{\mathrm{K}}$ (and $T_{0}$ ). In support of this basic understanding, we observe that the $T_{0}$ value obtained for the bulk using the total intensity of the $E_{\mathrm{F}}$ peak and its CEF sideband (curve $(2+3)$ in Fig. $3 d$ ) is $\sim 116 \mathrm{~K}$, which is larger than the one obtained for the surface $\sim 86 \mathrm{~K}$ and is consistent with the expected effect of a reduced $\frac{V^{2}}{\epsilon_{f}}$ on the surface for constant $N_{f}$. Indeed, for the case of SO sideband features in SIAM, it was noticed long $\mathrm{ago}^{29}$ that, although there is no rigorous proof, numerical calculations indicate that the total weight of the near $E_{\mathrm{F}}$ resonance, including its SO sideband, is roughly the same as would occur if the SO splitting were zero with no other change in the parameters, in which case $T_{\mathrm{K}}$ would be much larger and all the weight would lie in the $E_{\mathrm{F}}$ peak. This property should be essentially the same for the CEF sidebands.

The argumentation of the first case nicely reconciles the basic finding of a larger characteristic temperature for the $E_{\mathrm{F}}$ peak of the surface spectrum than for that of the bulk. But we can argue for a second, stronger and more interesting limiting case as follows. Working in the framework of the SIAM Cornut and Coqblin ${ }^{30}$ showed that as increasing $T$ successively populates excited CEF states, the effective $T_{\mathrm{K}}$ is successively increased as $N_{f}$ is successively increased. Since the CEF splittings all lie within the $T$ range of our measurements, one might expect fairly abrupt changes in the peak shape or temperature dependence to accompany such jumps in $T_{\mathrm{K}}$. However, as Fig. 3a, c shows, neither of these changes can be observed and the temperature dependence looks qualitatively similar to that of the bulk, where no CEF states become occupied in the measured temperature range. This suggests that the CEF splittings could be as small as the lower limit of our $T$ range, $\sim 1 \mathrm{meV}$, in which case, over the $T$ range of our measurements, the CEF splittings can be entirely neglected so that the ground-state degeneracy is effectively $N_{f}=6$. That the CEF splittings could be so small is consistent with our argumentation above for the role of the changed symmetry on the surface. In this case, there will be a significant $T$ range for which the surface magnetic moment remains Kondo quenched well after the bulk magnetic moments have begun to manifest. To our knowledge, such a difference in bulk and surface magnetic behavior has not been proposed or evidenced previously.

Finally, we note that besides the degeneracy $N_{f}$, the Kondo temperature further depends on the density of the itinerant states at $E_{\mathrm{F}}$ to which the local $4 f$ states can couple. Indeed, a much larger density for the Ce-terminated surface would have the same effect as the increase of the degeneracy $N_{f}$. However, while one might expect small differences, our measurements as well as ab initio band-structure calculations do not point to any strong discrepancies in the density of states at $E_{\mathrm{F}}$ for the two terminations. Therefore, we do not believe that the density of states could be the origin of the unexpected characteristic temperature relations.

\section{Dispersion of the Kondo peak}

Our second major finding concerns a large difference in the dispersion of the Kondo peak for surface and bulk, arising from the rather different surface and bulk itinerant states to which the $4 f$ states couple. To study this effect, the ARPES data must be interrogated for the $4 f$ dispersion above $E_{\mathrm{F}}$, where the difference is especially large. For this task, an analysis method was required and devised that allows for a further extend above $E_{\mathrm{F}}$ than the validity range of $5 k_{\mathrm{B}} T^{9}$ of the usual technique of dividing the data by the (resolution-broadened) FDD function. We assume that the observed intensity reduction of the $E_{\mathrm{F}}$ peak around the $\bar{\Gamma}$ point is caused by a dispersion of the Kondo resonance to higher energies. As described in the Supplementary Discussion 5, we deduce the necessary energy shifts by detailed modeling of the measured ARPES spectra in a small angle range for a number of different emission angles $\Phi$ at multiple temperatures $T$. As shown below the results obtained are consistent with an effective hybridization model, providing ex post facto confidence in the method. Although this method is tailored to the situation at hand, it should be more generally applicable.

Figure $4 a, b$ shows the deduced dispersion of the Kondo peak above $E_{\mathrm{F}}$ for the Ce and Si terminations, respectively. As we can see, the dispersion of the Kondo peak for the Ce-terminated surface is relatively weak, its position varies between 10 and $20 \mathrm{meV}$ above $E_{\mathrm{F}}$. On the other hand, for the Si-terminated surface, 

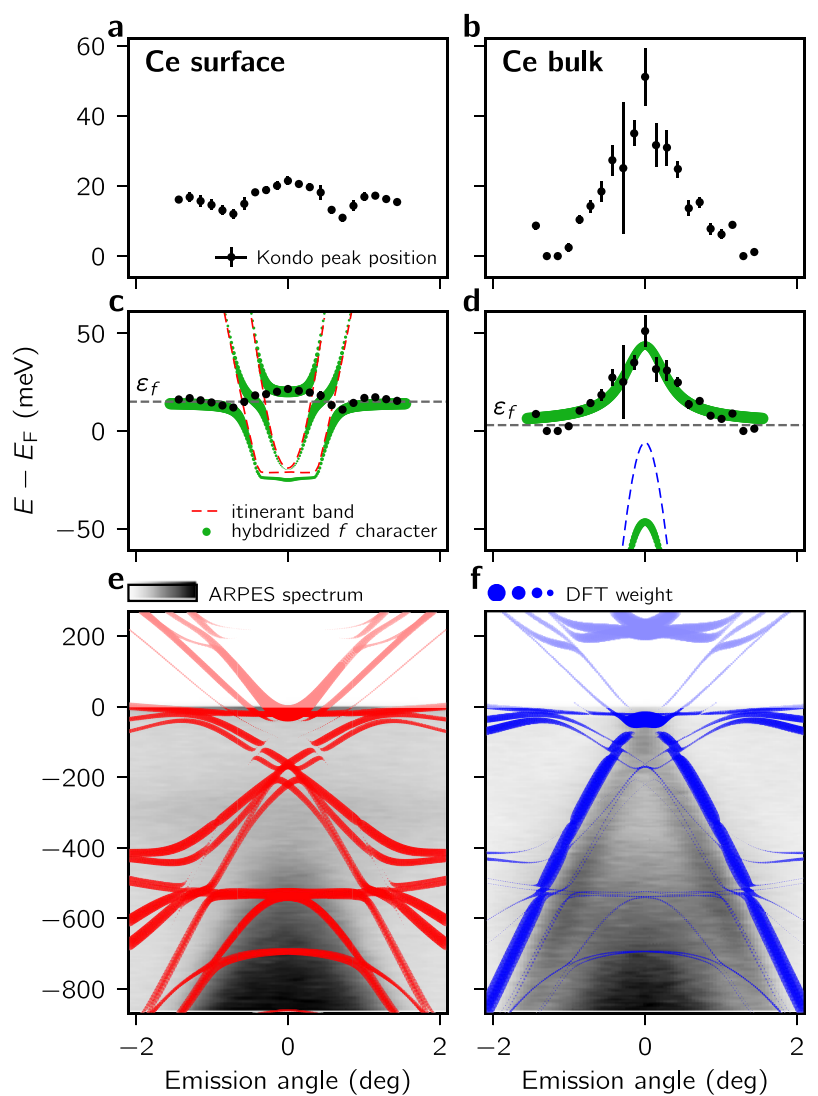

Fig. 4 Kondo peak dispersion. Dispersion of the Kondo peak above $E_{\mathrm{F}}$ near the $\overline{\bar{\Gamma}}$ point derived at $T=21 \mathrm{~K}$ for $\mathbf{a} \mathrm{Ce}$ - and $\mathbf{b}$ Si-terminated surfaces of $\mathrm{CeRh}_{2} \mathrm{Si}_{2}$. Respective hybridization models with c two electron-like bands $\left(V=10 \mathrm{meV}, \varepsilon_{f}=15 \mathrm{meV}\right)$ and $\mathbf{d}$ one hole-like band $\left(V=40 \mathrm{meV}, \varepsilon_{f}=3 \mathrm{meV}\right)$ with the unhybridized bands shown as dashed lines and the $f$ character of the hybridized bands shown in green as well as the results of band-structure calculations for e Ceand $f$ Si-terminated surfaces. The itinerant bands allowed for hybridization with $\mathrm{Ce}-4 f$ states are shown in red (surface $\mathrm{Ce}$ ) and blue (bulk-like $\mathrm{Ce}$ ). Note that the calculated bands are slightly shifted upward $(55 \mathrm{meV})$ in order to coincide with the ARPES data taken off-resonance $(h v=112 \mathrm{eV})$ and shown as gray-scale background (for an individual comparison of DFT and ARPES data see Supplementary Discussion 4).

it is seen that the position of the bulk Kondo peak is changed strongly from close proximity to $E_{\mathrm{F}}$ up to nearly $50 \mathrm{meV}$ above $E_{\mathrm{F}}$, forming a cone around the $\bar{\Gamma}$ point. To find the reason for this behavior, we used an effective hybridization model, where a localized $4 f$ state at an effective energy $\varepsilon_{f}$ slightly above $E_{\mathrm{F}}$ interacts with itinerant states taken from ab initio band-structure calculations. The obtained results are shown in Fig. 4c, d which can describe the determined dispersions nicely.

For the Si-terminated surface, the apex of the hole-like band (Fig. $4 \mathrm{f}$ ) is pinned to $E_{\mathrm{F}}$ at the $\bar{\Gamma}$ point. A strong hybridization of $4 f$ states with this band pushes the Kondo peak above $E_{\mathrm{F}}$ and leads to its strong $k$ dependence. For the Ce-terminated surface, however, there is no such explicit, and highly dispersive itinerant state in the vicinity of $E_{\mathrm{F}}$. There, the respective hole-like band is shifted to higher $\mathrm{BE}$ due to charge transfer. Instead, the hybridization takes place with a few electron-like bands leading to a moderate dispersion of the Kondo peak. Note that a similar behavior was observed for the heavy-fermion compound $\mathrm{YbRh}_{2} \mathrm{Si}_{2}$, where the Kondo resonance lies below $E_{\mathrm{F}}{ }^{31}$.

The rather different dispersion of the Kondo resonance for surface and bulk Ce might give insight into a more general problem of a Kondo lattice with two different local-moment sublattices. This problem was theoretically studied recently ${ }^{32}$, indicating a complex behavior with either competing or cooperative Kondo effects depending on the model parameters. However, our data show no clear evidence for an interplay between the different Kondo effects at the $\mathrm{Ce}$ in the bulk and the $\mathrm{Ce}$ on the surface. On the contrary, the hybridization of different itinerant bands to the $4 f$ states should prevent a strong direct cross talk of the Kondo effect on the different sites. A similar situation might prevail in compounds with different Ce sublattices, for instance in the case of the heavy-fermion systems $\mathrm{Ce}_{3} \mathrm{Pdln}_{11}{ }^{33}$ and $\mathrm{Ce}_{3} \mathrm{PtIn}_{11}{ }^{34}$, where it is thought that one of the crystallographic $\mathrm{Ce}$ sites is responsible for AFM ordering while the second one is responsible for superconductivity. Hence this could be one of the reasons why clear evidence for a cross talk of Kondo effects in such systems is yet lacking.

\section{Concluding remarks}

We have presented the results of $T$-dependent VUV-ARPES measurements on the AFM Kondo lattice $\mathrm{CeRh}_{2} \mathrm{Si}_{2}$. We have explored the Ce- $4 f$ spectral pattern taken from $\mathrm{Ce}$ - and $\mathrm{Si}$ terminated surfaces, which reflect the properties of $\mathrm{Ce}$ atoms at the surface and in the bulk, respectively. It was shown that the $4 f$ states near $E_{\mathrm{F}}$ behave indeed rather differently for surface and bulk Ce systems with a larger characteristic temperature at the surface than in the bulk although an opposite behavior is expected due to the reduced coordination and thus hybridization at the surface.

Our results derive from the observation that, relative to the bulk, the CEF splitting at the surface is greatly reduced to be less than the experimental resolution. At the simplest level, the $E_{\mathrm{F}}$ peak of the surface spectrum then includes both the Kondo resonance and the CEF sidebands, which implies an increase of the effective uf degeneracy $N_{f}$ that more than compensates the smaller hybridization at the surface. This understanding is strongly supported by the observation that adding to the bulk Kondo peak the contribution of its clearly separated sideband, a higher characteristic temperature is obtained for the bulk than for the surface. Because the temperature dependences of the spectra and their intensities show no hint of the successive jump increases in $T_{\mathrm{K}}$ that should arise as CEF states are successively thermally populated, we further infer that the CEF splittings are actually less than our lowest measured $T \sim 1 \mathrm{meV}$. This implies that for a considerable temperature range the surface magnetic moments are quenched even though the moments are manifested in the bulk, a possibility never proposed or evidenced before.

Our here applied and possibly generalizable analysis method allowed us further to visualize and discuss the $k$ dependences of the Kondo peak considerably far above $E_{\mathrm{F}}$ for both surface and bulk $\mathrm{Ce}$, which also differ considerably. This distinct behavior is caused by a considerable difference in the underlying surface and bulk valence-band structures to which the Ce- $4 f$ states couple.

Our findings show the possibility of disentangling the different surface and bulk contributions to the Kondo scale at a high level of detail, and may also provide an important clue in the better understanding of the Kondo problem when including a CEF term. Thereby our study brings into reach the ultimate goal of quantitatively testing many-body theories that link spectroscopy and transport properties, for both the bulk and the surface, separately. It also allows for a direct insight into the broader problem of Kondo lattices with two different local-moment sublattices, providing some understanding of why the crosstalking between the two Kondo effects is weak. 


\section{METHODS}

Details of the experiment

ARPES experiments were performed at the 105-beamline of the Diamond Light Source facility equipped with a ScientaR4000 analyzer. The samples of $\mathrm{CeRh} \mathrm{Si}_{2}$ were cleaved in situ under ultra-high vacuum conditions better than $10^{-10} \mathrm{mbar}$ at a temperature of $200 \mathrm{~K}$. At this temperature, the sample was characterized and two sample positions for the different surface terminations determined. In the following $T$-dependent measurements the temperature was varied from $142 \mathrm{~K}$ down to $10 \mathrm{~K}$ and the different surface terminations were sequentially measured at each temperature to ensure exactly the same conditions for both terminations. During the measurement, the optics of the beamline were not changed making certain that the same set-up applies to all spectra. The measurements were performed at the $\mathrm{Ce}$ $4 d \rightarrow 4 f$ absorption threshold at a photon energy of $121 \mathrm{eV}$ to emphasize the If contributions from the valence-band emission. The typical angular resolution was $0.2^{\circ}$ with an overall energy resolution of around $10 \mathrm{meV}$.

\section{Electronic structure calculations}

The ab initio band-structure calculations were performed in the framework of DFT within the local density approximation (LDA) using the Fullpotential nonorthogonal local-orbital minimum-basis band-structure scheme (FPLO) ${ }^{35}$. To account for the electronic structure of the Ce- and Si-terminated surface an asymmetric slab of 16 atomic layers was built on the basis of experimental data for lattice parameters and atomic positions. Surface relaxation effects along the surface normal were taken into account for the four outermost layers on the $\mathrm{Ce}$ - and Si-terminated side of the slab, respectively. Ce- $4 f$ states were moved from the valence basis to the core (open core approximation). The occupancy of the non-polarized $4 f$ shell was set to unity. For comparison with the experimental results, $E_{\mathrm{F}}$ of the computed bands was shifted by $55 \mathrm{meV}$.

\section{DATA AVAILABILITY}

All relevant data are available from the corresponding authors upon request.

Received: 19 March 2020; Accepted: 8 September 2020;

Published online: 06 October 2020

\section{REFERENCES}

1. Stewart, G. R. Heavy-fermion systems. Rev. Mod. Phys. 56, 755-787 (1984).

2. Stewart, G. R. Non-Fermi-liquid behavior in $d$ - and f-electron metals. Rev. Mod. Phys. 73, 797-855 (2001).

3. Riseborough, P. S. \& Lawrence, J. M. Mixed valent metals. Rep. Prog. Phys. 79, 084501 (2016).

4. Lawrence, J. M., Riseborough, P. S. \& Parks, R. D. Valence fluctuation phenomena. Rep. Prog. Phys. 44, 1-84 (1981).

5. Steglich, F. et al. Superconductivity in the presence of strong Pauli paramagnetism: $\mathrm{CeCu}_{2} \mathrm{Si}_{2}$. Phys. Rev. Lett. 43, 1892-1896 (1979).

6. Si, Q. \& Steglich, F. Heavy fermions and quantum phase transitions. Science $\mathbf{3 2 9}$, 1161-1166 (2010).

7. Allen, J. W. The Kondo resonance in electron spectroscopy. J. Phys. Soc. Japan 74, 34-48 (2005).

8. Reinert, F. et al. Temperature dependence of the Kondo resonance and its satellites in $\mathrm{CeCu}_{2} \mathrm{Si}_{2}$. Phys. Rev. Lett. 87, 106401 (2001).

9. Ehm, D. et al. High-resolution photoemission study on low- $T_{\mathrm{K}}$ Ce systems: Kondo resonance, crystal field structures, and their temperature dependence. Phys. Rev. B 76, 045117 (2007).

10. Patil, S. et al. ARPES view on surface and bulk hybridization phenomena in the antiferromagnetic Kondo lattice $\mathrm{CeRh}_{2} \mathrm{Si}_{2}$. Nat. Commun. 7, 11029 (2016).

11. Danzenbächer, S. et al. Wave-vector dependent intensity variations of the Kondo peak in photoemission from CePd $\mathrm{C}_{3}$. Phys. Rev. B 72, 033104 (2005).

12. Laubschat, C., Kaindl, G., Schneider, W.-D., Reihl, B. \& Mårtensson, N. Stability of $4 f$ configurations in rare-earth-metal compounds. Phys. Rev. B 33, 6675-6683 (1986).

13. Laubschat, C. et al. Surface electronic structure of a-like Ce compounds. Phys. Rev. Lett. 65, 1639-1642 (1990)

14. Vyalikh, D. V. et al. Wave-vector conservation upon hybridization of $4 f$ and valence-band states observed in photoemission spectra of a Ce monolayer on W (110). Phys. Rev. Lett. 96, 026404 (2006).

15. Mulazzi, M., Shimada, K., Jiang, J., Iwasawa, H. \& Reinert, F. Evidence of coexisting Kondo screening and valence fluctuations in the $\mathrm{CePd}_{7} / \mathrm{Pd}(001)$ surface alloy. Phys. Rev. B 89, 205134 (2014).
16. Kucherenko, Y., Molodtsov, S. L., Heber, M. \& Laubschat, C. $4 f$-derived electronic structure at the surface and in the bulk of $a$-Ce metal. Phys. Rev. B 66, 155116 (2002).

17. Laubschat, C., Weschke, E., Domke, M., Simmons, C. \& Kaindl, G. Surface and bulk electronic structure of $a$-Ce metal and Celr 2 . Surf. Sci. 269/270, 605-609 (1992).

18. Boursier, $\mathrm{R}$. et al. Comparison between $\mathrm{Ce}$ and $\mathrm{Yb}$ heavy fermion compounds: $\mathrm{CeRh}_{2} \mathrm{Si}_{2}$ versus $\mathrm{YbRh}_{2} \mathrm{Si}_{2}$. Physica B 403, 726-730 (2008).

19. Grier, B. H., Lawrence, J. M., Murgai, V. \& Parks, R. D. Magnetic ordering in $\mathrm{CeM}_{2} \mathrm{Si}_{2}$ $(M=A g, A u, P d, R h)$ compounds as studied by neutron diffraction. Phys. Rev. B 29, 2664-2672 (1984).

20. Kawarazaki, S. et al. Ground-state magnetic structure of $\mathrm{CeRh}_{2} \mathrm{Si}_{2}$ and the response to hydrostatic pressure as studied by neutron diffraction. Phys. Rev. $B$ 61, 4167-4173 (2000).

21. Graf, $\mathrm{T}$. et al. Magnetic phase transitions in $\mathrm{CeRh}_{2} \mathrm{Si}_{2}$ : specific heat, susceptibility, and resistance studies. Phys. Rev. B 57, 7442-7445 (1998).

22. Kucherenko, Y. et al. Giant hybridization effects in $4 f$ photoemission spectra of $\mathrm{Pr}$ and Nd transition-metal compounds. Phys. Rev. B 65, 165119 (2002).

23. Laubschat, C., Grentz, W. \& Kaindl, G. $4 f$ multiplets in bremsstrahlung-isochromat spectroscopy as a probe for local electron density in Ce compounds. Phys. Rev. $B$ 36, 8233-8236 (1987).

24. Willers, T. et al. Spectroscopic determination of crystal-field levels in $\mathrm{CeRh}_{2} \mathrm{Si}_{2}$ and $\mathrm{CeRu}_{2} \mathrm{Si}_{2}$ and of the $4 f^{0}$ contributions in $\mathrm{CeM}_{2} \mathrm{Si}_{2}(M=\mathrm{Cu}, \mathrm{Ru}, \mathrm{Rh}, \mathrm{Pd}$, and $\mathrm{Au})$. Phys. Rev. B 85, 035117 (2012).

25. Amorese, A. et al. Crystal electric field in $\mathrm{CeRh}_{2} \mathrm{Si}_{2}$ studied with high-resolution resonant inelastic soft x-ray scattering. Phys. Rev. B 97, 245130 (2018).

26. Holder, M. G. et al. CeFePO: $f-d$ hybridization and quenching of superconductivity. Phys. Rev. Lett. 104, 096402 (2010).

27. Uchima, K., Shigeoka, T. \& Uwatoko, Y. Magnetic characteristics of polymorphic single crystal compounds $\mathrm{Dylr}_{2} \mathrm{Si}_{2}$. Physica B 536, 28-33 (2018).

28. Jang, S. et al. Evolution of the Kondo lattice electronic structure above the transport coherence temperature. PNAS, https://doi.org/10.1073/pnas.2001778117 (2020).

29. Allen, J. W. et al. Electronic structure of cerium and light rare-earth intermetallics. Adv. Phys. 35, 275-316 (1986).

30. Cornut, B. \& Coqblin, B. Influence of the crystalline field on the Kondo effect of alloys and compounds with Cerium impurities. Phys. Rev. B 5, 4541-4561 (1972).

31. Vyalikh, D. V. et al. $k$ dependence of the crystal-field splittings of $4 f$ states in rareearth systems. Phys. Rev. Lett. 105, 237601 (2010).

32. Benlagra, A., Fritz, L. \& Vojta, M. Kondo lattices with inequivalent local moments: competitive versus cooperative Kondo screening. Phys. Rev. B 84, 075126 (2011).

33. Kratochvílová, M. et al. Coexistence of antiferromagnetism and superconductivity in heavy fermion Cerium compound $\mathrm{Ce}_{3} \mathrm{Pdln}_{11}$. Sci. Rep. 5, 15904 (2015).

34. Prokleška, J., Kratochvílová, M., Uhlírová, K., Sechovský, V. \& Custers, J. Magnetism, superconductivity, and quantum criticality in the multisite cerium heavy-fermion compound $\mathrm{Ce}_{3}$ PtIn 11 . Phys. Rev. B 92, 161114 (2015).

35. Koepernik, K. \& Eschrig, H. Full-potential nonorthogonal local-orbital minimumbasis band-structure scheme. Phys. Rev. B 59, 1743-1757 (1999).

\section{ACKNOWLEDGEMENTS}

This work was supported by the German Research Foundation (DFG) through Grants No. KR3831/5-1, No. LA655/20-1, Fermi-NEst, GRK1621, and SFB1143 (project-id 247310070). We also acknowledge support from the Spanish Ministry of Economy (MAT-2017-88374-P), the Russian Foundation for Basic Research (Grant No. 20-3270127), and Saint Petersburg State University (Grant No. ID 51126254). We acknowledge Diamond Light Source for access to beamline 105 (proposals no. SI17761-1 and SI24339-1) where the here presented data were measured. The research leading to this result has been supported by the project CALIPSOplus under Grant Agreement 730872 from the EU Framework Programme for Research and Innovation HORIZON 2020. Open Access funding enabled and organized by Projekt DEAL.

\section{AUTHOR CONTRIBUTIONS}

D.V.V. designed the research; N.C.-C. and C.G. grew and characterized the samples; G.P., S.S., M.M., M.G., A.G., S.D., and D.V.V. performed the UV-ARPES experiments; operation of the UV-ARPES facility was carried out by P.D. and A.V.F.; data analysis was performed by G.P. and the obtained results were discussed together with M.G., C.G., C.K., K.KI., and K.Ku.; the manuscript was written by G.P., Yu.K., J.W.A., C.L., and D.V.V.

\section{COMPETING INTERESTS}

The authors declare no competing interests. 


\section{ADDITIONAL INFORMATION}

Supplementary information is available for this paper at https://doi.org/10.1038/ s41535-020-00273-7.

Correspondence and requests for materials should be addressed to G.P. or D.V.V.

Reprints and permission information is available at http://www.nature.com/ reprints

Publisher's note Springer Nature remains neutral with regard to jurisdictional claims in published maps and institutional affiliations.
Open Access This article is licensed under a Creative Commons Attribution 4.0 International License, which permits use, sharing, adaptation, distribution and reproduction in any medium or format, as long as you give appropriate credit to the original author(s) and the source, provide a link to the Creative Commons license, and indicate if changes were made. The images or other third party material in this article are included in the article's Creative Commons license, unless indicated otherwise in a credit line to the material. If material is not included in the article's Creative Commons license and your intended use is not permitted by statutory regulation or exceeds the permitted use, you will need to obtain permission directly from the copyright holder. To view a copy of this license, visit http://creativecommons. org/licenses/by/4.0/.

(c) The Author(s) 2020 